\title{
Design of Ant-Inspired Stochastic Control Policies for Collective Transport by Robotic Swarms
}

\author{
Sean Wilson - Theodore P. Pavlic · Ganesh \\ P. Kumar · Aurélie Buffin · Stephen C. Pratt . \\ Spring Berman
}

the date of receipt and acceptance should be inserted later

\begin{abstract}
In this paper, we present an approach to designing decentralized robot control policies that mimic certain microscopic and macroscopic behaviors of ants performing collective transport tasks. In prior work, we used a stochastic hybrid system model to characterize the observed team dynamics of ant group retrieval of a rigid load. We have also used macroscopic population dynamic models to design enzyme-inspired stochastic control policies that allocate a robotic swarm around multiple boundaries in a way that is robust to environmental variations. Here, we build on this prior work to synthesize stochastic robot attachment-detachment policies for tasks in which a robotic swarm must achieve non-uniform spatial distributions around multiple loads and transport them at a constant velocity. Three methods are presented for designing robot control policies that replicate the steady-state distributions, transient dynamics, and fluxes between states that we have observed in ant populations during group retrieval. The equilibrium population matching method (EPMM) can be used to achieve a desired transport team composition as quickly as possible; the transient matching method (TMM) can control the transient population dynamics of the team while driving it to the desired composition; and the rate matching method (RMM) regulates the rates at which robots join and leave a load during transport. We validate our model predictions in an agent-based simulation, verify that each controller design method produces successful transport of a load at a regulated velocity, and compare the advantages and disadvantages of each method.
\end{abstract}

Keywords collective transport; bio-inspired robotics; stochastic robotics; stochastic hybrid system; distributed robotic system

Sean Wilson · Spring Berman

School for Engineering of Matter, Transport and Energy, Arizona State University, Tempe, AZ, USA, Sean.T.Wilson@asu.edu, Spring.Berman@asu.edu

Theodore P. Pavlic · Aurélie Buffin · Stephen C. Pratt

School of Life Sciences, Arizona State University, Tempe, AZ, USA, tpavlic@asu.edu, baurelie@asu.edu, Stephen.Pratt@asu.edu

Ganesh P. Kumar

School for Computing, Informatics, and Decision Systems Engineering, Arizona State University, Tempe, AZ, USA, Ganesh.P.Kumar@asu.edu 


\section{Introduction}

Cooperative manipulation and transport of heavy payloads will be required in various potential swarm robotic applications, including automated construction, manufacturing, and warehouses; disaster response and search-and-rescue missions; assembly of ships and aircraft; and manipulation, assembly, and construction tasks in inhospitable space and marine environments. In such applications, robots will be tasked to form a team around a payload and coordinate their motion and applied forces to transport the load to a predefined destination. Although various approaches to this task have been developed, there remains a need for a rigorous swarm control framework that can produce reliable transport in a wide range of scenarios with arbitrary payloads, unstructured and possibly hazardous environments, and lack of prior information about the loads and environment. Toward this end, we address the problem of developing a control framework for collective transport that is scalable with the number of robots, robust to robot failures and communications degradation, and agnostic to prior data and global position information. The control framework is reliant on a central supervisor only for high-level directives, and it is amenable to analysis, control, and optimization techniques that accommodate stochastic robot behaviors such as random encounters with environmental features.

Group food retrieval in ants (Fig. 1) is a valuable source of design inspiration for multirobot transport. This phenomenon is a striking example of a cooperative manipulation strategy that is (a) fully decentralized and scalable in the number of transporters, (b) conducted without specialized end effectors, and (c) successful for a wide range of payloads in environments with uneven terrain and obstacles. The behavioral mechanisms underlying group retrieval remain poorly understood (Czaczkes and Ratnieks 2013; McCreery and Breed 2013), but coordination likely depends on indirect interactions through the load itself, known as stigmergy (Grassé 1959; Kube and Bonabeau 2000), although more direct interactions and signaling among transporters may play a role as well. Coordination also depends on some or all of the ants knowing the heading to the nest and being able to maintain this direction using visual or other cues. In our prior work (Kumar et al 2013), we fit a stochastic hybrid system (SHS) model to experimental time-series data on group retrieval by the desert ant Aphaenogaster cockerelli. Although the model describes the data well, it does not prescribe stochastic policies that may be used in the control of multi-robot transport teams. In this paper, we use the SHS ant model as a reference trajectory generator in the design of communication-free, encounter-based robot behaviors that achieve ant-like collective transport performance. Our approach makes use of our previous work (Pavlic et al 2013) on an enzyme-inspired boundary coverage strategy that allocates a robotic swarm among stationary region boundaries in target team sizes that are robust to environmental variations, including the number and sizes of regions. More recently, we have shown that this strategy can be implemented in scenarios with multiple types of regions, no robot communication, and a specified convergence rate to the target equilibrium team sizes (Pavlic et al 2014). In summary, this paper extends our previous work on boundary coverage to scenarios in which the regions to be covered are mobile payloads that must be transported at a certain velocity, the robots must attain nonuniform spatial distributions around these loads at equilibrium, and the robot controllers are derived from experimentally based models of eusocial insect behavior.

We consider scenarios in which there are loads of multiple types that need to be moved from one location to another along linear trajectories. Each type of load requires a different robot team size for safe transport. We assume that the robots can differentiate between load types, but they have no prior information on the load quantities and locations. The robots execute random walks throughout the environment and rely on local sensor information to 


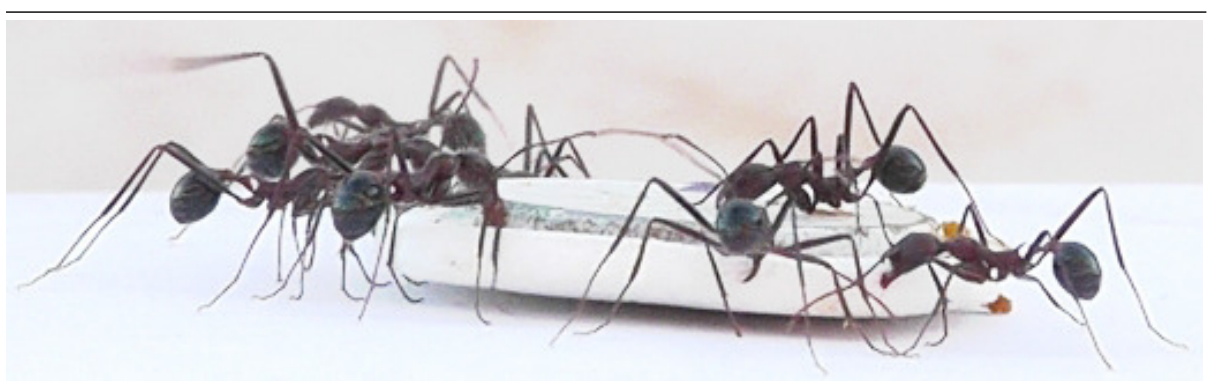

Fig. 1 Image from Kumar et al (2013) of a team of Aphaenogaster cockerelli ants carrying a weighted circular foam disk along a leftward direction. The ants on the left ("Front") side of the disk are assumed to be lifting and pulling the object, while the ants on the right ("Back") side of the disk are assumed to be only lifting the object. For size and weight reference, the object on top of the foam load is a U.S. dime $(2.3 \mathrm{~g}, 1.8 \mathrm{~cm}$ diameter).

trigger their pre-programmed control policies. We consider robot densities that are below levels at which robot crowding severely impedes robot motion and load transport. We develop three methods of designing the robot control policies to mimic ant behaviors that are effective at forming and maintaining transport teams. The equilibrium population matching method (EPMM) produces steady-state transport teams that match the observed ant distributions around a load. The transient matching method (TMM) reproduces the transient population dynamics of the ants during group retrieval. Finally, the rate matching method (RMM) replicates the fluxes of ants between sections of a load boundary. Using NetLogo (Wilensky 1999) simulations of an agent-based model of multi-robot transport scenarios, we demonstrate that our controller design methods can successfully reproduce the population dynamics that are predicted by our SHS model of group transport in A. cockerelli.

The paper is organized as follows. In Sect. 2, we define our ant-inspired transport scenario and develop a control architecture that can be implemented on each individual robot. Then, in Sect. 3, we describe the three controller design methods that mimic different microscopic and macroscopic properties of collective transport in ants. Section 4 outlines our simulation setup, discusses the simulation results, and summarizes the advantages and disadvantages of the controller design methods. In Sect. 5, we compare our approach to other control strategies for swarm robotic systems and relate it to the process of adsorption, a natural surface phenomenon where atoms or molecules adhere to a surface. Finally, we give concluding remarks in Sect. 6.

\section{Control Strategies for Robots to Achieve Non-uniform Distributions around Loads}

This paper focuses on the ant-inspired cooperative transport problem summarized in Fig. 2. The problem is to design stochastic control policies for individual robots that, when implemented on a sufficiently large swarm, will produce desired allocations of robots around each load and maintain those team sizes while the load is being carried toward its destination. In our previous work on boundary coverage regulation (Pavlic et al 2013, 2014), we designed robot attachment-detachment strategies for allocating target-sized populations that are uniformly randomly distributed around regions at equilibrium. Here, we show how that approach can be extended to achieve non-uniform distributions of robots around loads. In particular, we seek to match the observation (Kumar et al 2013) that more ants accumulate on the leading side of a transported object and appear to interact differently with the 


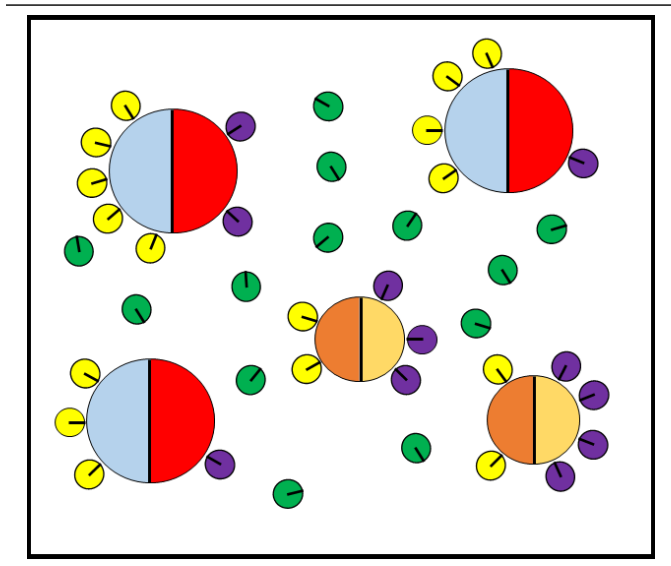

(a) Multi-robot transport arena

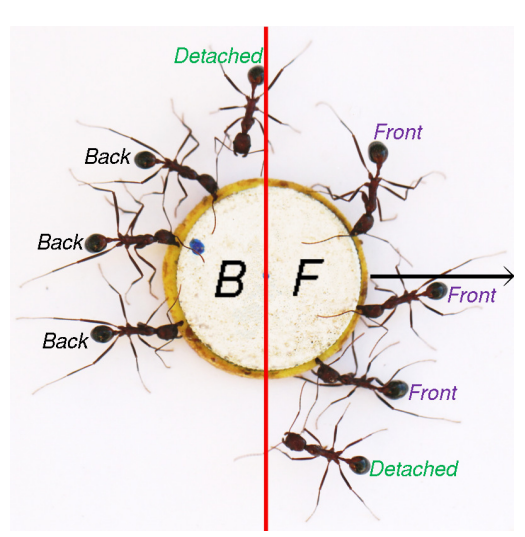

(b) Top view of ant transport team

Fig. 2 Multi-robot and multi-ant collective-transport scenarios. In (a), an arena is depicted with five loads surrounded by a swarm of robots, including some that have attached to the loads and some that are moving freely between the loads. The three large loads are of type 1, and the two smaller loads are of type 2. Although the size of a load may not be observable by an individual robot, the load type is assumed to be measurable (e.g., by the color of the load or its surface texture). The loads are divided into a Back (left) half and a Front (right) half. Based on the type of the object, a certain number of robots is desired on each of the two halves. Robots bound to the Back (left) halves of the loads are shown in yellow, robots bound to the Front (right) halves are shown in purple, and the freely moving Detached robots are shown in green. This scenario is based on the observations of ants in (b) from Kumar et al (2013). There, the circular load is moving to the right, and ants are characterized as grasping the Back (left) or Front (right) of the load; otherwise, they are Detached ants.

load based on their attachment position with respect to the direction of motion. As this is a stochastic allocation strategy, the equilibrium distributions will be dynamic: there will be continual fluctuations above and below the desired allocation levels, but the mean allocations will converge to these levels. Moreover, as long as there are enough robots available to reach the desired allocation levels on the loads, but not so many that robot crowding impedes individual robot motion and load transport, the equilibrium mean allocation levels will be insensitive to the size of the swarm and the density of robots in the arena.

\subsection{Robot Controller Architecture}

The robot controller that we design is illustrated by the state-transition diagram in Fig. 3. As we discuss elsewhere for the application of boundary coverage (Pavlic et al 2014), the catalytic detachment process that we use allows allocation policies for multiple load types to be decoupled. Consequently, without loss of generality, we consider only one type of load here, which we define as disk-shaped. Although there is only one type of load, there are two types of subregions: the leading (Front) and trailing (Back) sides of the load with respect to its transport direction (Fig. 2(b)). We define an unbound zone as a load sector with an arc length equal to the linear distance that a robot can occupy along the load perimeter. A bound zone is comprised of a robot attached to the load along with the adjacent load sector.

Matching our description of the ant behaviors, we specify that robots switch between Front, Back, and Detached states. Each robot is initially Detached and executes a correlated random walk (CRW) in order for the population to disperse approximately uniformly 
throughout the arena. In a CRW, robots iterate through short straight paths that are each punctuated by a turn to a random angle. However, other motion patterns that achieve similar dispersal are also valid choices. When a Detached robot encounters another robot, it executes maneuvers to avoid a collision. Alternatively, when a Detached robot encounters an unbound zone on the Front (or Back) of a load, it attaches with probability $p_{b F}$ (or $p_{b B}$ ). If a robot is attached to the Front (or Back) of a load and encounters a Detached robot nearby, the attached robot will detach with probability $p_{u F}\left(\right.$ or $p_{u B}$ ). In the absence of encounters, an attached robot will never detach from a load. In summary, the four parameters that characterize the attachment-detachment policy of each robot are:

- $p_{b F}$, the probability to attach (bind) to an encountered unbound zone on the Front of a load

- $p_{u F}$, the probability to detach (unbind) from a zone on the Front of a load after encountering a Detached robot

- $p_{b B}$, the probability to attach (bind) to an encountered unbound zone on the Back of a load

- $p_{u B}$, the probability to detach (unbind) from a zone on the Back of a load after encountering a Detached robot

Once attached to a load, a robot lifts with a small force $F_{\ell}$. A Front robot will additionally pull in the desired direction with a time-varying force described by the control law $K\left(v_{L}^{d}-\right.$ $\left.v_{L}(t)\right)$, where $K$ is a proportional gain, $v_{L}^{d}$ is a set point for the load velocity, and $v_{L}(t)$ is the measured load velocity at time $t$. Thus, robots have three additional parameters that characterize the transport of the load:

- $F_{\ell}$, the lifting force exerted by a robot that is attached to a load

$-v_{L}^{d}$, the desired velocity of the load

- $K$, the proportionality constant between the pulling force of a robot attached to the Front of a load and the error between the load velocity and $v_{L}^{d}$

\subsection{Load Dynamical Model}

As in our earlier work (Kumar et al 2013), we formulate the load dynamics using a double-integrator model that describes the relationship between the time-varying force inputs applied by the robots and the load acceleration. The model is fully characterized by two parameters:

- $m_{L}$, the mass of the load

$-\mu$, the kinetic coefficient of linear sliding friction

To prevent robots from switching directly between the Back to Front states due to load reorientation, we assume that rotational friction is sufficiently high to prevent load rotation. Even with the possibility of rotation, theoretical models of fixed teams of robots (Rubenstein et al 2013) show that rotation is only transient. Moreover, observational evidence of ants and simulated ant models (Berman et al 2011; Kumar et al 2013) show very little load rotation once smooth persistent load transport has begun. Thus, between attachment and detachment events, we model the time evolution of the load position $x_{L}$ and load velocity $v_{L}$ as linear translational motion:

$$
\left\{\begin{aligned}
\dot{x}_{L} & =v_{L} \\
m_{L} \dot{v}_{L} & =\eta_{F} K\left(v_{L}^{d}-v_{L}\right)-\mu\left(m_{L} g-\left(\eta_{F}+\eta_{B}\right) F_{\ell}\right)
\end{aligned}\right.
$$

where $\eta_{F}$ denotes the number of Front robots and $\eta_{B}$ denotes the number of Back robots. 


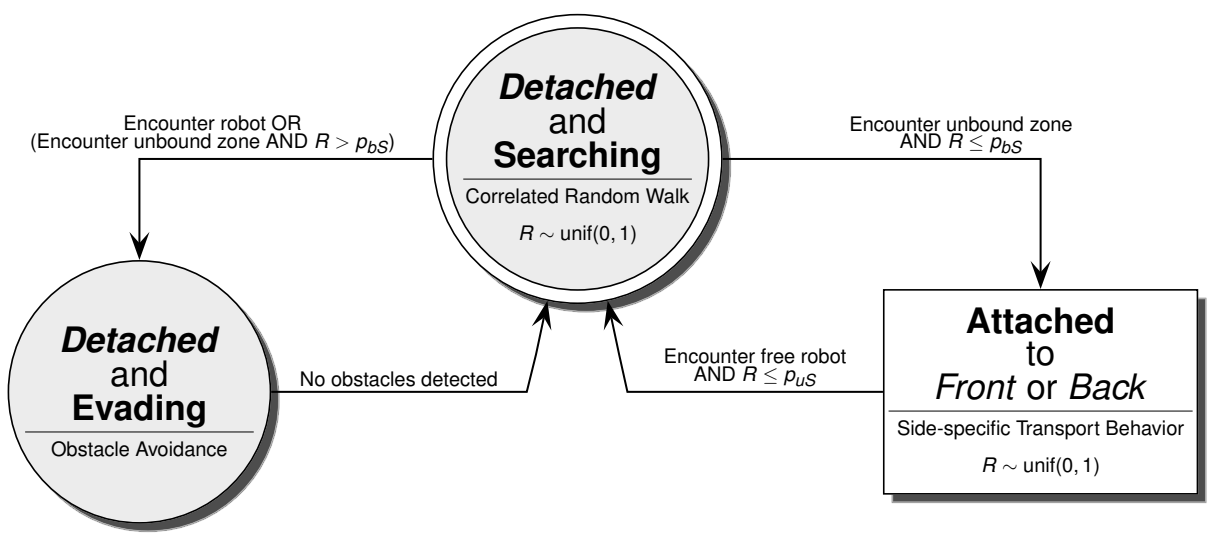

Fig. 3 State-transition diagram of robot controller for collective transport. The diagram outlines a program that would run on a single robot. The states shown as circles represent Detached robots that are unbound and free to move. The state shown as a rectangle represents either Front or Back robots that are attached to the leading or trailing edge of a load with respect to its motion and executing a side-specific transport behavior (e.g., Front robots lift and pull, and Back robots only lift). The Detached robots execute a spacefilling random motion primitive, such as a correlated random walk, and avoid obstacles as necessary. The robots employ a simple obstacle-avoidance algorithm: if a robot senses an object that it will not attach to, then it chooses another direction until it does not sense any interference. The subscript $S \in\{F, B\}$ represents the identified side of the load, Front or Back.

\section{Robot Controller Design for Mimicking Ant Behaviors during Collective Transport}

In our experimental work with A. cockerelli (Kumar et al 2013), we obtained data on the mean transport team dynamics and fit an SHS model in which ants switch between Back, Front, and Detached states at constant rates that signify probabilities per unit time. The averaged time-series data and the fitted model are plotted in Fig. 4, which shows the six per-ant state transition rates $\left(r_{B F}, r_{D F}, r_{F B}, r_{D B}, r_{F D}, r_{B D}\right)$ in the titles of the graphs in the top row. The state-transition system parameterized by these six rates is a Markov process on a fully connected graph. Thus, the time evolution of the states is described by a thirdorder linear time-invariant (LTI) system, which consists of three coupled linear ordinary differential equations with constant coefficients. The system converges at an exponential rate to the following mean numbers of Front and Back ants at equilibrium:

$$
[\text { Front }]^{*} \approx 5.78 \text { ants and }[\text { Back }]^{*} \approx 3.54 \text { ants with time constant } \tau \approx 52.08 \mathrm{~s} \text {. }
$$

This section describes three controller design methods for swarm-robotic mimicry of different aspects of the collective-transport behavior exhibited by the ants. In Sect. 3.1, we introduce the equilibrium population matching method (EPMM), which reproduces the average steady-state Front and Back populations from the ant data in the multi-robot scenario but does not necessarily reproduce the transient population dynamics. In Sect. 3.3, we introduce the transient matching method (TMM), which shows how the transient population dynamics can also be controlled when the average distributions of environmental features around the swarm do not change. Finally, Sect. 3.4 describes the rate matching method (RMM), which reproduces the ant Back-Front transition rates rather than the equilibrium allocations or system convergence rate. Results are provided in Sect. 4.2 that compare the outcomes of using these different methods. 

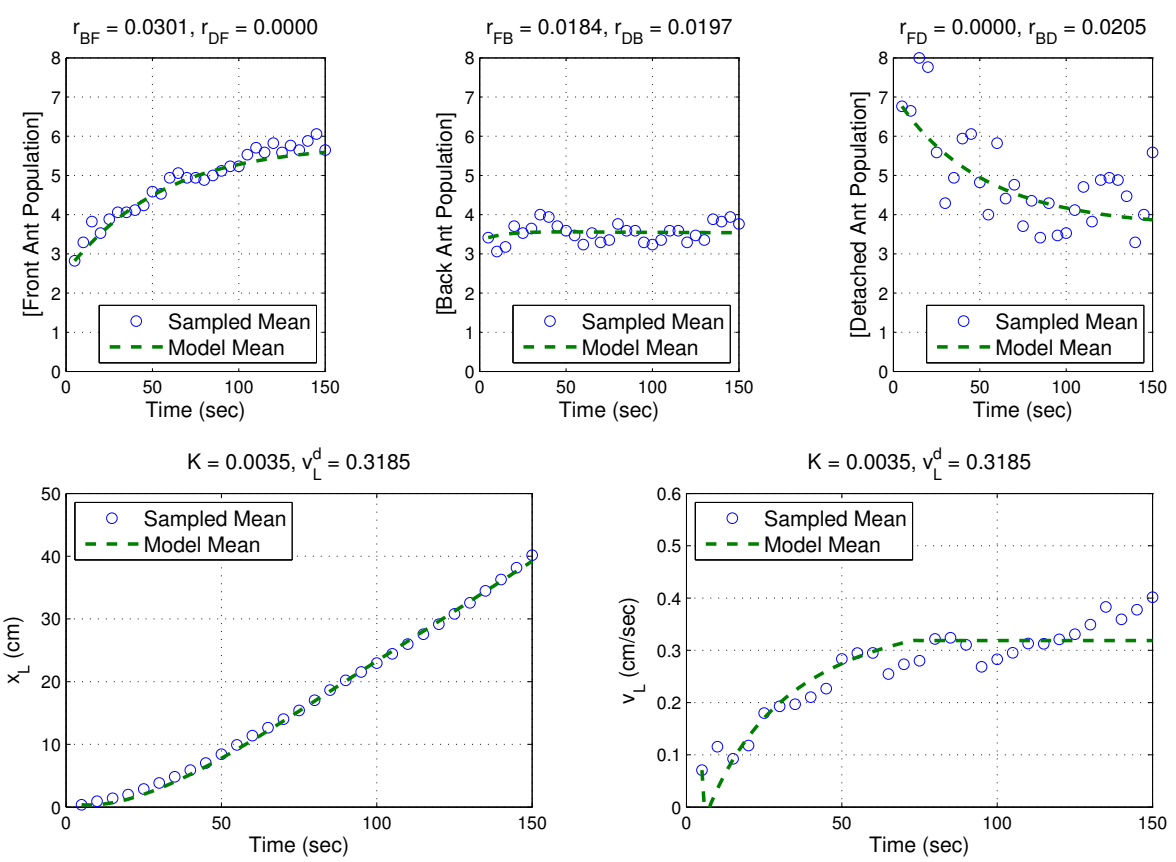

Fig. 4 Data from collective-transport experiments with A. cockerelli ants (Kumar et al 2013). The circles show the average of data from 17 experimental trials with small groups of $A$. cockerelli that carried a rigid circular load with mass $m_{L}=2.3 \mathrm{~g}$ and coefficient of sliding friction $\mu=0.58$. From left to right, the top row shows the mean numbers of Front, Back, and Detached ants over time. The bottom row shows the mean position (left) and velocity (right) of the load over time. The dashed line in each graph is the mean-field trajectory from an SHS model that has been fit to the data. From a separate experiment, the approximate mean lifting force of an individual ant was measured to be $F_{\ell}=2.653 \mathrm{mN}$. The free parameters in the model are the gain $K$, the velocity set point $v_{L}^{d}$, and the six rate constants $r_{S_{0} S_{1}}$ defining the per-ant transition rate from $S_{0} \in\{F, B, D\}$ to $S_{1} \in\{F, B, D\}$ with $S_{0} \neq S_{1}$. The best-fit values of these parameters are shown in the titles of the graphs.

In the EPMM, a control policy can be designed using predicted or experimentally determined values of the ratios of encounter rates, as opposed to the absolute values of these rates. These ratios will be invariant to changes in parameters such as robot density or speed, and so the equilibrium population distributions around loads will also be invariant to such changes. However, the transient dynamics of the system will be sensitive to changes in the absolute encounter rates. Thus, in both the TMM and RMM, the absolute encounter rates will need to be estimated in order to control the transient dynamics. Toward this end, Sect. 3.2 demonstrates how the EPMM can be used as a tool for inferring the encounter rates.

\subsection{Equilibrium Population Matching Method}

Here, we outline a modeling and control approach that simplifies the design of the attachment-detachment probabilities discussed in Sect. 2 so that we can guarantee that the mean Front and Back robot populations match those observed in the ant data shown in Fig. 4. Adapting our earlier work on stochastic boundary coverage (Pavlic et al 2013, 2014), we model the Detached-Front robot transitions and the Detached-Back robot transitions as 
two parallel chemical reaction networks (CRNs). The species in the CRNs are defined as $r$, a free (Detached) robot; $U_{F}\left(U_{B}\right)$, an unbound zone on the Front (Back) side of a load; and $B_{F}\left(B_{B}\right)$, a bound zone on the Front (Back) side of a load. We define $e_{u F}\left(e_{u B}\right)$ as the mean per-robot rate of encounters between a single free robot and a single unbound zone on the Front (Back) side of a load, and $e_{b F}\left(e_{b B}\right)$ as the mean per-robot rate of encounters between a single free robot and a robot that is attached to the Front (Back) side of a load. A reaction $r+U_{F} \stackrel{p_{b F} e_{u F}}{\longrightarrow} B_{F}$ signifies the following:

- The notation $r+U_{F}$ represents the event of a free robot $r$ encountering an unbound zone $U_{F}$ on the Front side of a load.

- The notation $\stackrel{p_{b F} e_{u F}}{\longrightarrow} B_{F}$ represents how often such encounter events occur and result in the free robot $r$ binding to the unbound zone $U_{F}$ to produce a new bound zone $B_{F}$.

The full CRN model is given by:

$$
\begin{array}{ll}
\underbrace{r+U_{F} \stackrel{p_{b F} e_{u F}}{\longrightarrow} B_{F}}_{\text {Front side }} & \text { and } \begin{array}{l}
r+U_{B} \stackrel{p_{b B} e_{u B}}{\longrightarrow} B_{B} \\
B_{F} \stackrel{p_{u F} e_{b F}}{\longrightarrow} U_{F}+2 r
\end{array}
\end{array} \quad \begin{aligned}
& \underbrace{r+B_{B} \frac{p_{u B} e_{b B}}{\longrightarrow} U_{B}+2 r}_{\text {Back side }}
\end{aligned} .
$$

Strictly speaking, the Front and Back CRNs are coupled by the shared pool of free robot $(r)$ reactants. However, for catalytic allocation to multiple task types, the equilibrium population for each type can be determined independently so long as there are more total robots than required for the desired equilibrium allocation (Pavlic et al 2014). So in this application, the equilibrium Front and Back populations are independent of each other so long as there are more total robots than the desired number of attached robots. Consequently, Front and Back control strategies can be designed independently. We denote the desired mean equilibrium quantities of unbound zones and bound zones on side $S \in\{F, B\}$ as $U_{S}^{*}$ and $B_{S}^{*}$, respectively, and define the target allocation ratio as $B_{F}^{*} /\left(U_{F}^{*}+B_{F}^{*}\right)$. For example, if we want the Front boundary of every load to be half covered with robots, then $U_{F}^{*}=B_{F}^{*}$ and the target allocation ratio is 0.5 .

Due to the fact that we are considering loads with a continuum of unbound zones (i.e., robots can attach anywhere on the loads), two attached robots on a load can have too little room between them to allow another robot to connect. This leads to a macro-scale model complication in which the presence of a unit of unbound space in the system does not necessarily imply that there is space available for further robot attachment. Consequently, the equilibrium of the well-mixed CRN model in Eq. (3) must be appropriately adjusted to account for this non-well-mixed effect (Pavlic et al 2013). Consider side $S \in\{F, B\}$ of the load and let $L_{S}$ be the arc length of the side normalized to the arc length that is occupied by an attached robot. That is, $\left\lfloor L_{S}\right\rfloor$ is the maximum number of robots that can attach to side $S$, and $L_{S}-\left\lfloor L_{S}\right\rfloor<1$ is the normalized arc length of the slack zone, the remaining fraction of an unbound zone. The relationship between the control pair $\left(p_{b S}, p_{u S}\right)$ and the target equilibrium bound-to-unbound zone ratio $B_{S}^{*} / U_{S}^{*}$ is:

$$
\frac{p_{b S}}{p_{u S}}=\frac{1+\delta_{S}^{*}}{1-\delta_{S}^{*} \frac{B_{S}^{*}}{U_{S}^{*}}} \frac{e_{b S}}{e_{u S}} \frac{B_{S}^{*}}{U_{S}^{*}} \quad \text { with } \quad \delta_{S}^{*} \triangleq \delta_{S}\left(\frac{B_{S}^{*}}{U_{S}^{*}+B_{S}^{*}}\right)
$$

where the function $\delta_{S}:[0,1] \mapsto[0,1]$ is given by

$$
\delta_{S}(r)=\frac{L_{S}}{\left\lceil L_{S}\right\rceil} \frac{\sin \left(\frac{2 \pi}{T_{S}}(1-r)\right)}{\sin \left(\frac{2 \pi}{T_{S}}\right)},
$$




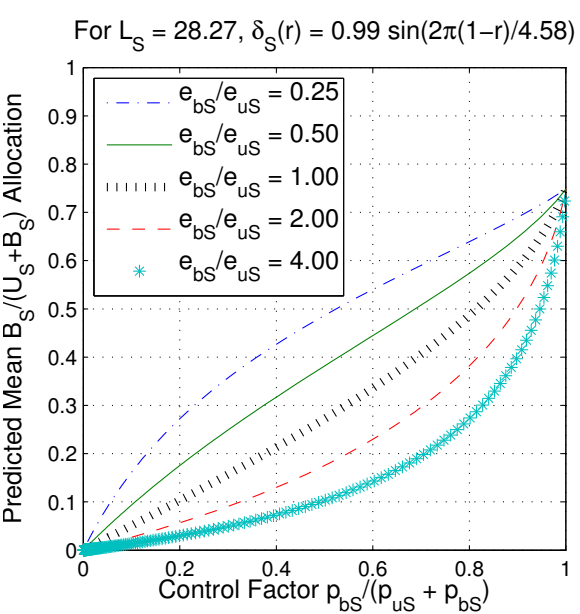

(a) $L_{S} \approx 30$

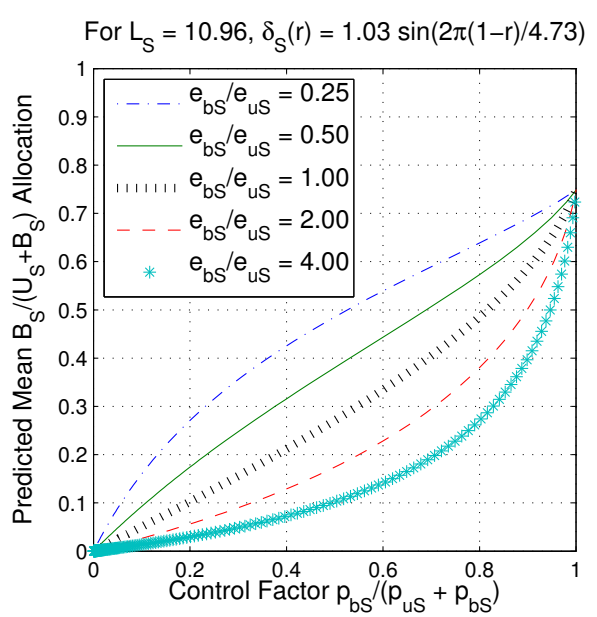

(b) $L_{S} \approx 10$

Fig. 5 Effect of encounter rate ratio on the relationship between the control factor $p_{b S} /\left(p_{u S}+p_{b S}\right)$ and the target equilibrium allocation ratio $B_{S}^{*} /\left(U_{S}^{*}+B_{S}^{*}\right)$ around side $S \in\{F, B\}$ (i.e., Front or Back), shown here for two different values of $L_{S}$ (Pavlic et al 2013). In general, each control factor has one degree of freedom in the $\left(p_{b S}, p_{u S}\right)$ space; however, here we let $p_{u S} \equiv 1$ for control factors less than 0.5 and $p_{b S} \equiv 1$ otherwise. Although these curves technically depend on the value of $L_{S}$, they are insensitive to small changes in this parameter. As predicted by analysis of classical random sequential adsorption (RSA) (Evans 1993; Rényi 1958), the equilibrium allocation for $\left(p_{b S}, p_{u S}\right)=(1,0)$ is the so-called parking constant (Solomon and Weiner 1986; Finch 2003) of approximately 0.75 . That is, robots that randomly attach to a load are unlikely to optimally pack. Each curve only depends on the ratio $e_{b S} / e_{u S}$ of the robot encounter rates with bound and unbound zones. Disturbances such as changes in robot density or speed are likely to change the two absolute encounter rates in similar ways. Consequently, this ratio is robust to such changes.

and the parameter $T_{S}$ is the largest value that ensures that $\delta_{S}(\gamma)=(1-\gamma) / \gamma$ for the parking constant $\gamma \approx 0.7476$ (Solomon and Weiner 1986; Finch 2003). The value of $\delta_{S}(r)$ represents the mean length of the unbound zones along sides $S$ as a function of the target allocation ratio. The extremes of this function are $\delta_{S}(1)=0$ and $\delta_{S}(0)=L_{S} /\left\lceil L_{S}\right\rceil \approx 1$. The quantity $\left\lceil L_{S}\right\rceil$ represents the total number of unbound zones, including the slack zone, on an empty side $S$. Thus, $L_{S} /\left\lceil L_{S}\right\rceil \approx 1$ is the average length of an unbound zone on an empty load. The parameter $T_{S}$ is fixed by the value of $L_{S} /\left\lceil L_{S}\right\rceil$ and can be solved for during the design process. In general, $T_{S} \in[4.1,4.77]$, which can be verified by solving for $T_{S}$ over a range of $L_{S} \in[1, \infty)$. When robots are very small relative to the load (i.e., $L_{S} \approx \infty$ ), then $T_{S} \approx 4.76$.

Equation (4) constitutes a control law that maps the target bound-to-unbound zone ratio, $B_{S}^{*} / U_{S}^{*}$, to the ratio of probabilities, $p_{b S} / p_{u S}$, which can be set by the control designer. Equivalently, Eq. (4) maps the target allocation ratio, $B_{S}^{*} /\left(U_{S}^{*}+B_{S}^{*}\right)$, to the ratio $p_{b S} /\left(p_{u S}+p_{b S}\right)$, which we call the control factor. Each control factor has an extra degree of freedom that can be used for optimization. For the EPMM, we set $p_{u S}=1$ for control factors less than 0.5 and $p_{b S}=1$ otherwise. This enforces a one-to-one mapping between control factors and $\left(p_{b S}, p_{u S}\right)$ pairs.

The relationship between control factors and allocation ratios is plotted in Fig. 5. The figure shows that for a given encounter rate ratio $e_{b S} / e_{u S}$, this relationship is described by a monotonically increasing curve that is anchored by zero on the left and the parking constant on the right. If it is easier for a robot to detect a bound zone than an unbound zone (e.g., due to the extra size that a bound robot adds to an unbound zone), then allocations will be lower 


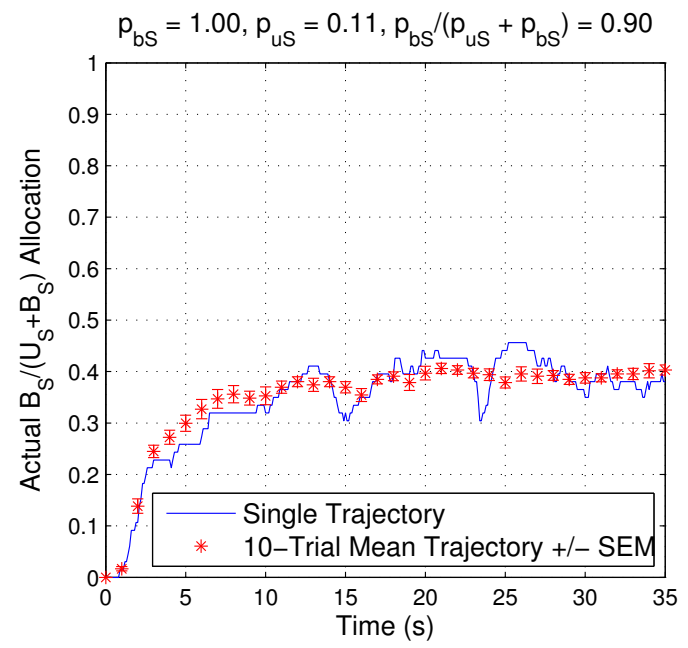

Fig. 6 Time evolution of the allocation ratio on a side $S \in\{F, B\}$ from simulation trials in NetLogo (for simulation details, see Sect. 4.1). The solid line shows the trajectory of the allocation ratio for a single trial when $\left(p_{b S}, p_{u S}\right)=(1,0.11)$, and the asterisks with error bars show the mean \pm one standard error of the mean (SEM) across ten trials with the same control pair. Each simulation used 300 initially unbound robots in an arena with three loads.

for the same control factor due to the increased frequency of robot-catalyzed unbinding. Conversely, if unbound zones are easier to detect than bound zones (e.g., because the motion primitive of recently unbound robots makes it very likely that they immediately re-encounter unbound zones), then the allocations will be higher for the same control factor. Hence, the relationship in Fig. 5 is governed by the motion and sensing characteristics of the robots and not by environmental parameters that can change over time, including the robot density and the number and sizes of loads. The encounter rate ratio can be estimated in two ways:

- If robots detaching from a load are no more likely to re-encounter that load than other robots in the nearby vicinity, then the ratio $e_{b S} / e_{u S}$ may be estimated as the ratio of the area of a bound zone to the area of an unbound zone. That is, for sides $S$ that are shaped like half-circles,

$$
\frac{e_{b S}}{e_{u S}} \approx \frac{\frac{\pi R^{2}}{2 M}+A}{\frac{\pi R^{2}}{2 M}}=1+\frac{2 A M}{\pi R^{2}},
$$

where $R$ is the radius of the load, $A$ is the area of a circular robot, and $M$ is the length of a side after normalizing to the arc length occupied by a single robot. However, it is often the case that robots that have recently detached from a load will have a higher encounter rate with unbound zones than robots that are randomly searching. Under these circumstances, estimation of the ratio $e_{b S} / e_{u S}$ would require the method described below.

- An empirical estimate of $e_{b S} / e_{u S}$ can be fit to a sampled version of Fig. 5 that consists of data from robot simulations or experimental trials in which the control factor is varied. For example, Fig. 6 shows the actual allocation ratio of a side $S$ over time for $\left(p_{b S}, p_{u S}\right)=$ $(1,0.11)$. The data were generated in NetLogo from simulations of 300 robots in an arena with three loads. The solid line plots the allocation ratio of the side over time during one trial. The asterisks show the mean allocation ratio across 10 trials, with bars 
showing one standard error of the mean. The mean converges to the allocation ratio $B_{S}^{*} /\left(U_{S}^{*}+B_{S}^{*}\right)$ corresponding to the control factor $p_{b S} /\left(p_{u S}+p_{b S}\right)=1 / 1.11=0.90$. In this particular case, the control factor 0.90 results in an equilibrium allocation ratio of 0.4. In Fig. 5, we find that the pair $(0.90,0.4)$ lies on the curve for $e_{b S} / e_{u S} \approx 4$. This process can be repeated for additional control factors to gain more confidence in the $e_{b S} / e_{u S}$ estimate.

\subsection{Using the EPMM to Estimate Absolute Encounter Rates}

As discussed at the beginning of Sect. 3, the TMM and RMM approaches described in Sects. 3.3 and 3.4 require the estimation of absolute encounter rates in order to precisely control the transient dynamics and transition rates of the robotic swarm. Analytical predictions of encounter rates based on geometry are not feasible for scenarios where robots are significantly smaller than loads, and estimating encounter rates from empirical data is typically very difficult (Hutchinson and Waser 2007; Gurarie 2008). There are typically unpredictable, non-linear effects from motion primitives of the robots and the non-uniformly randomly distributed spacing of attachment points on the loads through the environment. However, as shown in Sect. 3.1, swarms that follow the control policies encoded in the CRN Eq. (3) will converge in the mean to allocations which are only sensitive to the ratio $e_{b S} / e_{u S}$ of the encounter rates $e_{b s}$ and $e_{u s}$. Here, we discuss how measuring the convergence rate of a system designed using the EPMM allows for indirect inference of the encounter rates.

If the initial number $r_{0}$ of free robots is significantly larger than the number of robots needed to attain the desired allocation levels on the loads, then the likelihood of a free robot finding an unbound zone or bound zone will not vary over time. In other words, if the fraction of robots that are in the Detached state does not change appreciably over time, then the bimolecular CRNs in Eq. (3) can be replaced by reversible unimolecular CRNs:

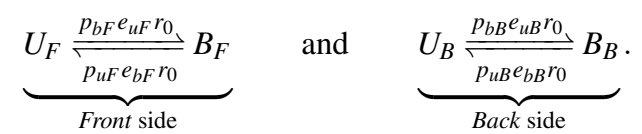

That is, because the system effectively has a buffered capacity of $r_{0}$ robots, the free robot species $r$ in Eq. (3) has been replaced with the scalar $r_{0}$, which is included in the rate constants in Eq. (6). So although all reactions in Eq. (3) are irreversible, they approximate the reversible system in Eq. (6). It is easy to show (Berman et al 2009; Odhner and Asada 2010) that the mean-field dynamics of a set of unimolecular reversible reactions are linear and time-invariant (LTI). In particular, for each side $S \in\{F, B\}$, the second-order LTI ordinary differential equation for the $U_{S} \rightleftharpoons B_{S} \mathrm{CRN}$ has two eigenvalues,

$$
\lambda_{0}=0 \quad \text { and } \quad \lambda_{1}=-\left(p_{b S} e_{u S} r_{0}+p_{u S} e_{b S} r_{0}\right),
$$

where $\lambda_{0}$ represents the conservation of the sum $U_{S}+B_{S}$, and $-\lambda_{1}$ is the sum of the two mass-action rates. Moreover, the time constant $\tau_{S}=-1 / \lambda_{1}$ for each side $S \in\{F, B\}$ is

$$
\begin{aligned}
\tau_{S} & =\frac{1}{\left(p_{b S} e_{u S}+p_{u S} e_{b S}\right) r_{0}} \\
& =\frac{1}{\left(p_{b S}+p_{u S} \frac{e_{b S}}{e_{u S}}\right) e_{u S} r_{0}}
\end{aligned}
$$




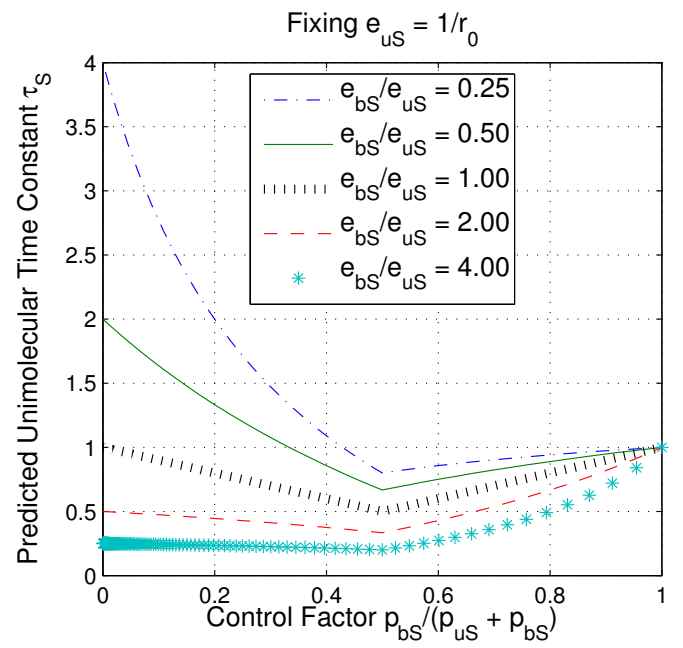

Fig. 7 Effect of encounter-rate ratio on the relationship between the control factor $p_{b S} /\left(p_{u S}+p_{b S}\right)$ and the approximate time constant $\tau_{S}$ for convergence to the equilibrium allocation on side $S \in\{F, B\}$ (Front or Back) (Pavlic et al 2014). The solution of a first-order linear time-invariant system (i.e., an exponential curve) can be fitted to the mean step response in Fig. 6 to predict the time constant $\tau_{S}$. Here, the predicted relationship between the control factor $p_{b S} /\left(p_{u S}+p_{b S}\right)$ and the time constant $\tau_{S}$ is shown, assuming that the system can be approximated as a unimolecular CRN. Although the actual convergence rate depends on the absolute encounter rates $e_{b S}$ and $e_{u S}$, the shape of the time constant curve is fixed by the encounter-rate ratio $e_{b S} / e_{u S}$. As in Fig. 5, we let let $p_{u S} \equiv 1$ for control factors less than 0.5 and $p_{b S} \equiv 1$ otherwise.

$$
\left.=\frac{1}{\left(\frac{p_{b S}}{p_{u S}}+\frac{e_{b S}}{e_{u S}}\right) e_{u S} p_{u S} r_{0}} \quad \stackrel{\text { (by Eq. }}{=}(4 \mathrm{a})\right) \quad \frac{U_{S}^{*}-\delta_{S}^{*} B_{S}^{*}}{U_{S}^{*}+B_{S}^{*}} \frac{1}{e_{b S} p_{u S} r_{0}}
$$

where three equivalent forms have been provided in Eq. (7) for application-specific convenience. In general, the relationship between $\tau_{S}$ and the control factor $p_{b S} /\left(p_{u S}+p_{b S}\right)$ depends on the absolute encounter rates $e_{u S}$ and $e_{b S}$. However, as shown in Eq. (7), the shape of the $\tau_{S}$ curve is fixed by the $e_{b S} / e_{u S}$ ratio and the absolute encounter rates only scale that relationship. Some example $\tau_{S}$ curves are shown in Fig. 7 for five different encounterrate ratios. Hence, for each of several $\left(p_{b S}, p_{u S}\right)$ pairs, a mean step response like Fig. 6 can be generated to determine the equilibrium allocation ratio $B_{S}^{*} /\left(U_{S}^{*}+B_{S}^{*}\right)$ and time constant $\tau_{S}$ for that pair. By the methods described at the end of Sect. 3.1, the allocation ratio data will determine the effective $e_{b S} / e_{u S}$ ratio. Then, Eq. (7) can be solved to yield an estimate of encounter rate $e_{u S}$ (and thus $e_{b S}$ as well). Using this method, encounter rates do not need to be known or solved for prior to simulating the system, but rather can be inferred from the observed behavior of the swarm.

Small-swarm case: If, initially, there are few free robots, the transient response to the sudden introduction of several unoccupied loads will be better described by Eq. (3) than Eq. (6). Moreover, the step response will rise with a logistic as opposed to an exponential shape (Pavlic et al 2013). However, if the resulting equilibrium allocations are perturbed by numbers of bound and unbound zones that are low enough to not appreciably change the number of free robots when the system restores equilibrium, then the transient response will be approximately exponential and the LTI-based encounter rate estimates above can still be applied. 


\subsection{Transient Matching Method}

In Sect. 3.1, we showed how the $p_{b S} / p_{u S}$ ratio can be chosen to achieve a desired bound-tounbound zone ratio $B_{S}^{*} / U_{S}^{*}$ at equilibrium on a side $S \in\{F, B\}$. This control policy provides one degree of freedom to the designer. Specifically, there is a line of $\left(p_{b S}, p_{u S}\right)$ pairs that achieve the same $B_{S}^{*} / U_{S}^{*}$ ratio at equilibrium. Consequently, the control policy can be optimized over this space. In Sect. 3.2, we showed how convergence rate measurements can be used to infer the absolute encounter rates $e_{b S}$ and $e_{u S}$. Once these encounter rates are known, we can then choose the precise $\left(p_{b S}, p_{u S}\right)$ pair that most accurately reproduces the transient dynamics exhibited by an ant collective-transport team. In particular, the values of the robot swarm size $r_{0}$ and the probability $p_{u S}$ (or, equivalently, $p_{b S}$ ) can be chosen to guarantee that the system converges with a desired time constant.

Here, we assume that the swarm is sufficiently large to allow the use of the unimolecular approximation in Eq. (6) with the time constant in Eq. (7). For any desired bound-tounbound zone ratio $B_{S}^{*} / U_{S}^{*}$, Eq. (4) specifies the corresponding $p_{b S} / p_{u S}$ ratio. Moreover, Eq. (7c) provides an expression for the time constant $\tau_{S}$ with free parameters $e_{u S}, p_{u S}$, and $r_{0}$. As described in Sect. 3.2, the rate $e_{u S}$ can be inferred from the EPMM approach. So, if $\tau_{S}^{*}$ is a desired time constant, the product $r_{0} p_{u S}$ should be chosen so that $p_{u S} \in[0,1]$ and

$$
r_{0} p_{u S}=\frac{1}{\left(\frac{p_{b S}}{p_{u S}}+\frac{e_{b S}}{e_{u S}}\right) e_{u S} \tau_{S}^{*}} .
$$

For any swarm size $r_{0}$ and $p_{b S} / p_{u S}$ ratio, the probability $p_{u S}$ (or, equivalently, $p_{b S}$ ) can be chosen to scale the convergence rate, with the fastest convergence at $p_{u S}=1$ and very slow convergence at $p_{u S} \approx 0$. So, to achieve any time constant $\tau_{S}^{*}$, the number of robots $r_{0}$ must be sufficiently high so that $p_{u S}$ can then be used to scale the convergence rate to the desired value.

\subsection{Rate Matching Method}

In Sects. 3.1 and 3.3, we addressed how to achieve a desired equilibrium mean allocation at a desired convergence rate. Alternatively, there may be some applications where the priority is to match the fluxes of ants and robots switching between states, as opposed to their equilibrium allocation levels. Toward this end, we present a method for matching the FrontBack transition rates of the ant and robot systems.

Under the assumption of a relatively large robotic swarm with respect to the number of bound zones at equilibrium, we can approximate the robot state transitions as a set of two unimolecular reversible reactions like Eq. (6) with LTI dynamics. Because it is not physically possible in our system for a robot to directly transition from Back to Front and vice versa, transitions to an intermediate Detached state are necessary. Figure 8(a) shows a Markov chain representing the transitions that a robot can execute. The system has four rate constants: two for the Front-Detached transitions and two for the Back-Detached transitions. A complication arises from the fact that our ant data were sampled at 5-second intervals (Kumar et al 2013), and so some recorded ants appear to transition directly from one side of the load to the other. Because of this, our SHS model of the ant collective-transport dynamics has six rate constants, shown in the titles of the top plots in Fig. 4: the same four as in the multi-robot scenario $\left(r_{F D}, r_{D F}, r_{B D}, r_{D B}\right)$, plus two more for the Back-Front transitions $\left(r_{B F}, r_{F B}\right)$. The resulting Markov chain followed by the observed ants is shown 


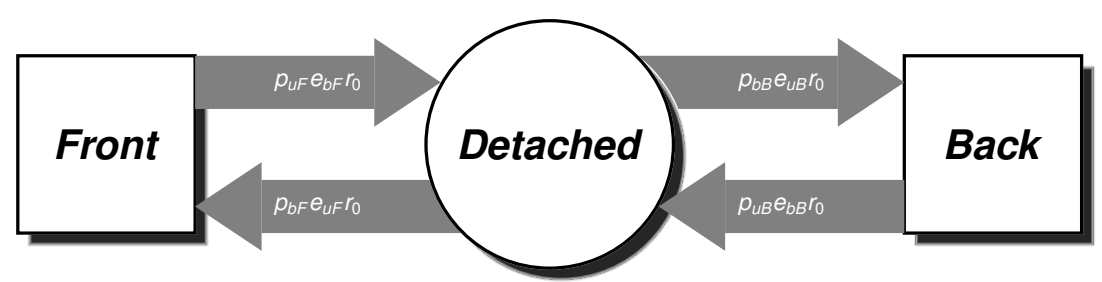

(a) Markov chain of implemented robot

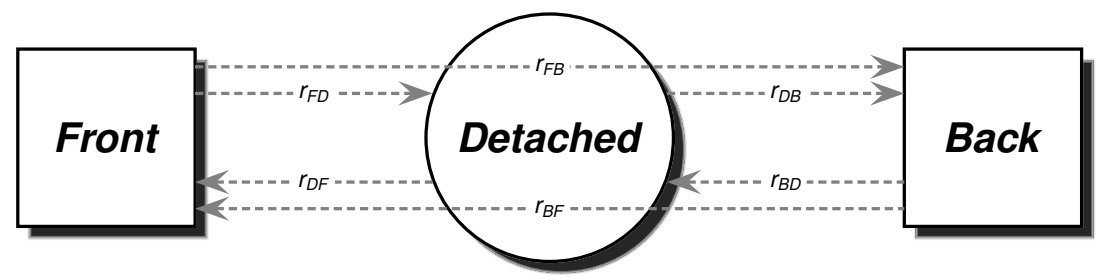

(b) Markov chain of sampled ant

Fig. 8 Single-ant and single-robot Markov chains. Each agent, ant or robot, transitions among Front, Detached, and Back states. In a robotic implementation with random attachment and catalytic detachment, direct transitions between Back and Front are not possible. Consequently, in (a), only four transition rates are shown. However, in data collected from ants, some ants appear to transition directly between the two attached states. Consequently, the model fit to the ant data introduces two additional transition rates, $r_{F B}$ and $r_{B F}$, which are shown in (b) as transitions cutting directly across the Detached state. To reconcile (a) and (b), the $r_{F B}$ rate can be added to both $r_{F D}$ and $r_{D B}$. Similarly, the $r_{B F}$ rate can be added to both $r_{B D}$ and $r_{D F}$. In other words, in order to observe a stable $r_{F B}$ (or, similarly, $r_{B F}$ ) rate in sampled data, there must be a hidden flux of Frontto-Detached ants that matches a flux of Detached-to-Back ants.

in Fig. 8(b). Comparing Figs. 8(a) and 8(b), it is clear that each direct transition from Front to Back could be alternatively recorded as a transition from Front to Detached followed by a transition from Detached to Back. Thus, we incorporate the $r_{F B}$ and $r_{B F}$ rates from the ant model into the robot control policies by adding them to the the Front-Detached and BackDetached rates in the multi-robot CRN:

$$
\underbrace{U_{F} \underset{r_{F D}+r_{F B}}{\stackrel{r_{D F}+r_{B F}}{\rightleftharpoons}} B_{F}}_{\text {Front side }} \text { and } \underbrace{U_{B} \frac{r_{D B}+r_{F B}}{r_{B D}+r_{B F}}}_{\text {Back side }} B_{B},
$$

Then, equating the rate constants in Eqs. (6) and (9) yields the programmed robot attachment-detachment probabilities

$$
\begin{aligned}
& p_{u F}=\frac{r_{F D}+r_{F B}}{e_{b F} r_{0}}, \quad p_{b B}=\frac{r_{D B}+r_{F B}}{e_{u B} r_{0}}, \\
& p_{u B}=\frac{r_{B D}+r_{B F}}{e_{b B} r_{0}}, \quad \text { and } \quad p_{b F}=\frac{r_{D F}+r_{B F}}{e_{u F} r_{0}}
\end{aligned}
$$

where $e_{b S}$ and $e_{u S}$ can be determined using the methods in Sect. 3.2 for each side $S \in\{F, B\}$. To ensure that these probabilities take values in the interval $[0,1]$, the swarm size $r_{0}$ must be chosen sufficiently large so that

$$
r_{0} \geq \max \left\{\frac{r_{F D}+r_{F B}}{e_{b F}}, \frac{r_{D B}+r_{F B}}{e_{u B}}, \frac{r_{B D}+r_{B F}}{e_{b B}}, \frac{r_{D F}+r_{B F}}{e_{u F}}\right\}
$$


Expected RMM results and caveats: Under the conditions above, the robotic swarm will converge to the Front and Back allocation ratios

and

$$
\frac{B_{F}^{*}}{U_{F}^{*}+B_{F}^{*}}=\frac{1}{1+\delta_{F}^{*}} \frac{r_{D F}+r_{B F}}{r_{F D}+r_{F B}+r_{D F}+r_{B F}}
$$

$$
\frac{B_{B}^{*}}{U_{B}^{*}+B_{B}^{*}}=\frac{1}{1+\delta_{B}^{*}} \frac{r_{D B}+r_{F B}}{r_{B D}+r_{B F}+r_{D B}+r_{F B}},
$$

which can be derived from the combination of Eqs. (10) and (4). Moreover, from the combination of Eqs. (10) and (7a), the system will converge with Front and Back time constants

and

$$
\tau_{F}=\frac{1}{r_{F D}+r_{F B}+r_{D F}+r_{B F}}
$$

$$
\tau_{B}=\frac{1}{r_{B D}+r_{B F}+r_{D B}+r_{F B}} .
$$

Although the RMM is the most direct attempt at replicating the microscopic behaviors of the ants on robotic platforms, it is the least likely to match macroscopic transport properties such as equilibrium team sizes and convergence rates. At a basic level, there are zero degrees of freedom in Eqs. (11) and (12). Thus, the goal of the RMM to match rate constants across systems eliminates any ability to control the equilibrium of the system or its convergence rate. Moreover, fundamental differences between the SHS ant model and the multi-robot stochastic implementation make equilibrium or convergence-rate matching only possible by unlikely coincidence. The unimolecular CRN used to model the populations dynamics of the multi-robot system relies on the assumption of a sufficiently large swarm size $r_{0}$ that is buffered against changes due to robot attachments and detachments. However, the ant population sizes associated with the SHS model data were on par with the eventual transport team sizes. Consequently, the number of free ants was appreciably decreased as teams formed around loads, and thus the attachment rate was limited by the fewer available free ants for further attachment. Therefore, it is generally expected that the TMM will produce a closer match in the population dynamics than the RMM when the reference ant swarm is small. The TMM allows for implementation-level dissimilarities in order to better match desired macroscopic characteristics.

Although the TMM will better match macroscopic allocation properties, there can be causes where fluxes from one region to another are more relevant than coverage around regions. In these cases, an RMM-like approach may be more appropriate. For example, consider a case like the one described by Zhang et al (2013) where robots have been programmed with an algorithm to track the shapes, sizes, and movements of puddles in an area by skirting the perimeter of each puddle. Similar to our collective-transport problems, the boundaries of the puddles are analogous to the boundaries of the loads with the robots "attaching" to their boundaries in each case. If a central server is using swarm robots to find these puddles and sample their continuous time evolution, having a high concentration of robots on the boundaries corresponds to having a high spatial resolution of each sample. However, it may instead be of critical importance to return frequently enough to the central location to meet sampling-rate requirements (i.e., temporal resolution may be prioritized over spatial resolution). In this latter case, controlling the transition rate between the boundary and the central server allows for controlling the sampling rate. By using stochastic 


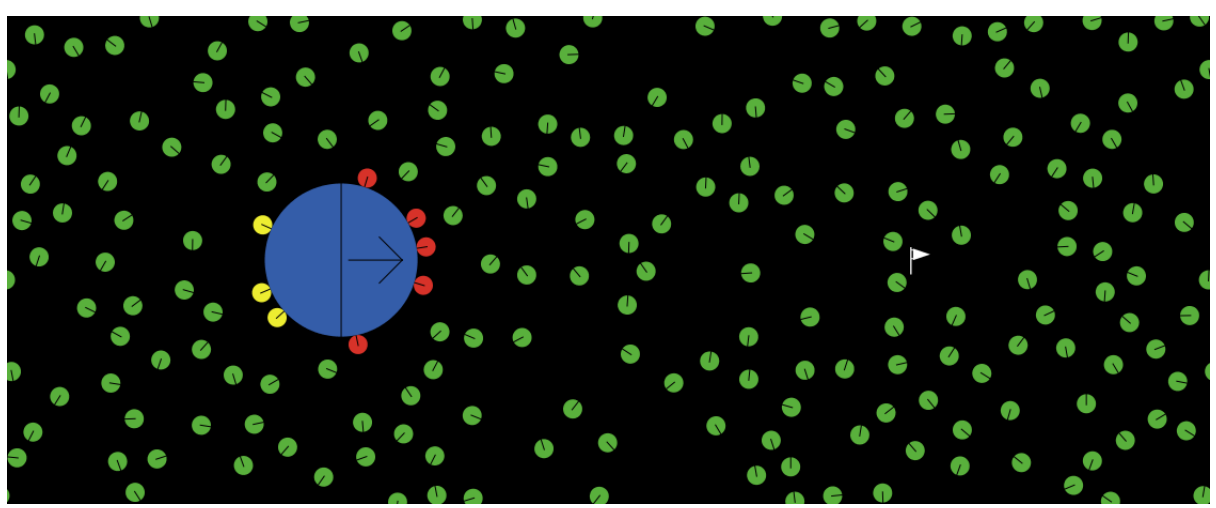

Fig. 9 A screenshot from the NetLogo simulation of collective transport. The large blue circle represents the load, red circles are Front robots, yellow circles are Back robots, and green circles are Detached robots. The arrow on the load indicates its direction of motion during transport, and the white flag indicates its goal position.

attachment-detachment policies designed by the RMM, ingress and egress fluxes (as opposed to boundary coverage at any given time) can be controlled, thus setting the sampling rate of the distributed swarm sensor system.

\section{Validation of Collective Transport Control Strategies in Simulation}

\subsection{Simulation Setup}

We tested the control policies computed by the EPMM, TMM, and RMM with simulations of multi-robot collective transport in the agent-based model simulation software NetLogo (Wilensky 1999). Each of these three methods generated a different set of attachment and detachment stochastic control policies, $\left(p_{b F}, p_{u F}\right)$ and $\left(p_{b B}, p_{u B}\right)$; all other parameters were held constant across the simulations. To demonstrate that the control policies are effective even with a single load, we considered the scenario depicted in Fig. 9. In the simulations, $r_{0}=200$ robots with radius $1 \mathrm{~cm}$ are initially placed randomly throughout an arena of size $125 \mathrm{~cm} \times 50 \mathrm{~cm}$. A rigid circular load with radius $8 \mathrm{~cm}$ and mass $2.3 \mathrm{~g}$ is placed in the arena with the robots. During the simulations, each robot runs the controller shown in Fig. 3 with one of the three sets of control policies. The Detached robots perform correlated random walks with a turning angle of $45^{\circ}$. Using our experimentally measured values of ant speeds and ant forces applied to a vision-based force sensor as guidelines (Berman et al 2011), we set the robot speed to $10 \mathrm{~cm} / \mathrm{s}$ and the maximum robot pulling force to $10.5 \mathrm{mN}$.

\subsection{Results}

Following the procedure discussed in Sect. 3, we used the EPMM, TMM, and RMM to design transport behaviors for individual robots that replicate different aspects of previously collected ant data (Kumar et al 2013). As described in Sect. 3.1 for the EPMM, the encounter rate ratio $e_{b S} / e_{u S}$ for each side $S \in\{F, B\}$ was estimated by running a multi-robot transport simulation with different values for the control factor $p_{b S} /\left(p_{u S}+p_{b S}\right)$. In principle, this calibration process need only be done for a single control factor. We use a range of control factor 


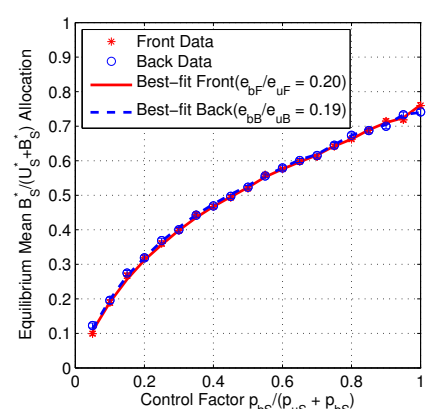

(a) Estimation of $e_{b S} / e_{u S}$ from equilibria.

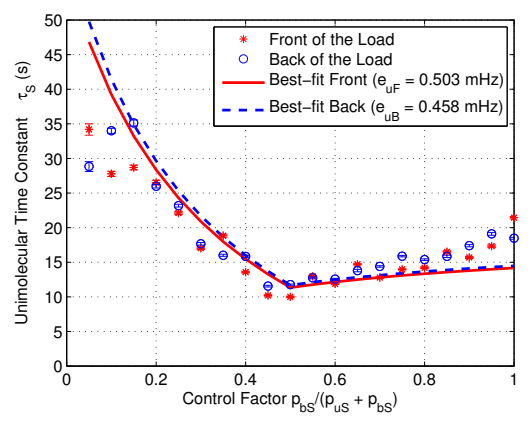

(b) Estimation of $e_{b S}, e_{u S}$ from convergence rates.

Fig. 10 Encounter rate estimation results. As in Fig. 6, a set of 100 identical multi-robot simulation trials were performed in NetLogo for each control factor value. Averaging across the set produced the Front and Back mean allocation ratios plotted in (a) with error bars showing standard error of the mean (SEM). The expected relationships shown in Fig. 5(b) were then fit to these empirical curves, yielding a $e_{b S} / e_{u S}$ ratio of approximately 0.2 for each side $S \in\{F, B\}$. The Front and Back time constants estimated from the average step response for each control factor value are plotted in (b). Using the $e_{b S} / e_{u S} \approx 0.20$ relationship from Fig. 7, these time constants were used to infer the encounter rates $e_{b S}$ and $e_{u S}$ for each side $S \in\{F, B\}$.

values here to show how well the microscopic multi-robot simulation matches macroscopic predictions. Like our ant experiments, our multi-robot simulations were run with a rigid circular load. Because the Front and Back sides of the loads have identical geometries, it is expected that the encounter rate ratios for both sides will be identical. Figure 10(a) confirms that this is indeed the case: $e_{b F} / e_{u F}=0.20$ and $e_{b B} / e_{u B}=0.19$.

For the TMM and RMM, the absolute encounter rates $e_{u S}$ and $e_{b S}$ must be estimated for sides $S \in\{F, B\}$ according to the procedure in Sect. 3.2. So, for each ensemble of trials used to generate Fig. 10(a), we also plotted average step responses similar to the one in Fig. 6 and estimated their rise time. The resulting time constants are shown in Fig. 10(b). Using the encounter rate ratios already estimated for the EPMM, we fit the relationships in Fig. 7 to these data to determine that $e_{u F}=0.503 \mathrm{mHz}$ and $e_{u B}=0.458 \mathrm{mHz}$, which could then be used with the encounter rate ratios to compute $e_{b F}$ and $e_{b B}$. These encounter rates are small because they represent the rate at which an individual robot (e.g., in an empty arena) would encounter a single specific zone of a load. This event happens rarely per robot, but the number of encounters that happen across a swarm is significantly larger.

Now equipped with $e_{b S} / e_{u S}, e_{b S}$, and $e_{u S}$ for each side $S \in\{F, B\}$, we applied Eqs. (4), $(8)$, and (10) to find the $\left(p_{b S}, p_{u S}\right)$ control pairs using the EPMM, TMM, and RMM. The simulation results are shown in Fig. 11 and are overlayed with the ant data, the SHS ant model trajectories, and the predicted equilibrium allocations. In all three cases, the mean equilibrium numbers of Front and Back robots are lower than the predicted values by less than 1 robot. This small discrepancy may be corrected by better estimating the $e_{b S} / e_{u S}$ encounter rate ratio. Moreover, because each side of the load can only support 12 attached robots that are optimally packed (eg. zero free space between attached robots on a load), quantization error can be a significant accuracy limitation in this scenario. For larger loads or smaller robots, more accurate mean allocations can be achieved. As shown by Figs. 11(a) and 11(b), the mean equilibrium allocations from the 100 EPMM simulations agree with those from the $100 \mathrm{TMM}$ simulations. Consequently, although the theoretical predictions can be slightly improved, the allocation results in general are highly repeatable. 

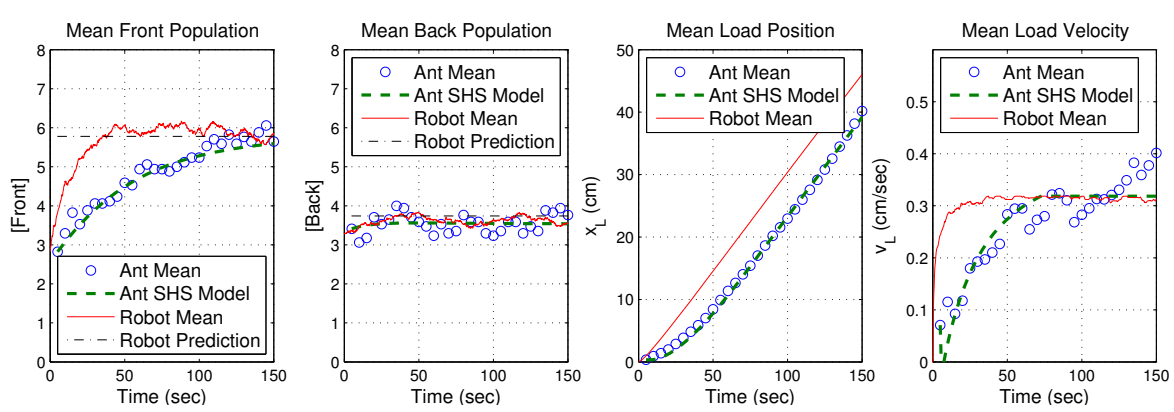

(a) Equilibrium Population Matching Method $\left(B_{F}^{*}=5.78, B_{B}^{*}=3.74\right.$; expected $\left.\tau_{F}=7.68 \mathrm{~s}, \tau_{B}=13.96 \mathrm{~s}\right)$
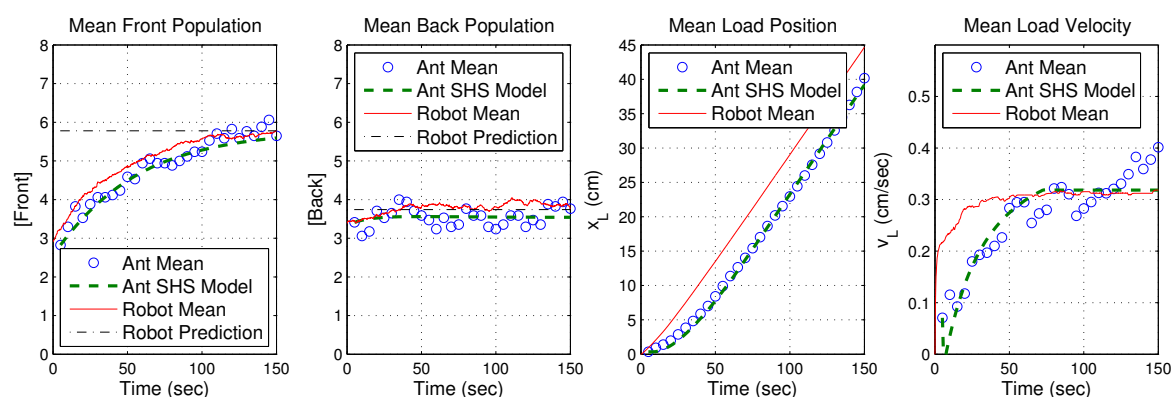

(b) Transient Matching Method $\left(\tau_{F}^{*}=\tau_{B}^{*}=52.08 \mathrm{~s}\right)$
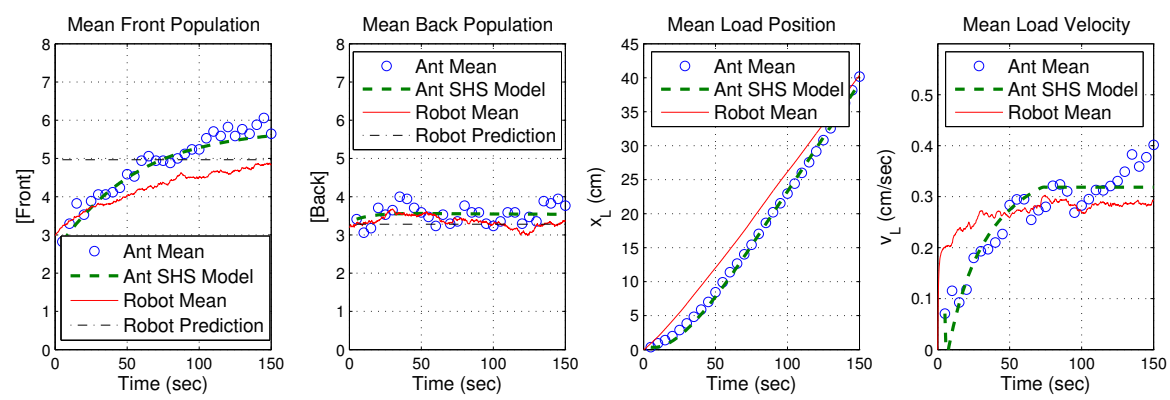

(c) Rate Matching Method (rates from Fig. 4; expected $B_{F}^{*}=5.09, B_{B}^{*}=3.35, \tau_{F}=20.62 \mathrm{~s}, \tau_{B}=11.27 \mathrm{~s}$ )

Fig. 11 Population dynamics and load position and velocity during collective transport by ants and robots. The plots show results from 100 simulated trials with robot control policies designed using each of the three methods described in Sect. 3. The left two columns show the time evolution of the mean numbers of Front and Back individuals; the right two columns show the trajectories of the mean position and speed of a single non-rotating load moving along a line. In each graph, the reference data from the ant trials are shown as circles, trajectories from the SHS model fit to the ant data are shown as dashed curves, and the multi-robot simulation data is shown as a solid line. The horizontal dash-dotted lines in the left two columns show the predicted equilibrium allocations based on the control approach.

\subsection{Discussion}

We have presented three methods of designing stochastic encounter-based controllers that drive robotic swarms to transport payloads along linear trajectories. In the EPMM used to generate Fig. 11(a), the extra degree of control freedom was used to maximize the convergence rate to equilibrium. As a result, the time constants $\tau_{F}$ and $\tau_{B}$ differ from those of 
the ant population dynamics, and the $\tau_{F}$ constant results in a much faster rise in the mean Front robot population than in the ant data. Because the transport team forms much faster, it achieves velocity regulation more quickly. Because of this, the position of the simulated load takes an early but bounded lead with respect to the position of the load in the ant data. These results show that the EPMM is most suited for cases where it is necessary to achieve steady transport of the load in the minimum possible amount of time. The EPMM control policies can produce rapid changes in the Front population, leading to quick fluctuations of the pulling force on the load and thus to a jerky start to the load's motion. However, the method guarantees the fastest convergence to the load velocity set point and target equilibrium allocations.

The TMM reduces the discrepancy between the transient dynamics of the ant and robot transport teams, as evidenced by the results in Fig. 11(b). The TMM results show the same equilibrium team sizes as the EPMM, as expected, but the rise time of the Front robot population more closely matches that of the Front ant population. Moreover, the rise time of the load speed is increased, and the lead of the load position with respect to the ant team's load trajectory is thereby decreased. However, although the TMM is able to better match the transient population dynamics of the ants, the load velocity still rises too quickly compared to the ant data. This disparity may be due to differences in how nonlinearities are implemented in the SHS and NetLogo friction models. In particular, the SHS model appears to show a delay before its initial rise that is not reflected in the NetLogo simulation. Another factor could be differences in the effectiveness and degree of coordination between the robot and ant transport teams. The simulated Front robots always pull the load in the desired direction with a force defined according to a prescribed control law. However, the ants could be counteracting each other's efforts by pulling in different directions, and they may not be exerting their maximum possible forces. Unlike the EPMM, the TMM requires information on the absolute encounter rates, but it allows for control over the transient population dynamics. This control can effect a slower growth of the transport team if desired, which can produce a smoother acceleration of the load.

Finally, the RMM results in Fig. 11(c) show that control design based on rate constant matching does not reproduce any macroscopic properties of the ant teams in the simulated multi-robot transport system. The RMM leaves no degrees of freedom to control the allocation ratio, and so the predicted allocation ratios differ from the EPMM and TMM cases. However, this method is useful when it is necessary to control the fluxes of robots that join and leave the load boundary. In the EPMM and TMM cases, there is no control over the frequency of attachment and detachment events that occur during transport. The RMM controls the rate at which these events occur and can be useful when frequent robot attachment and detachment is risky to the transport dynamics or to the object being transported (e.g., if the load is brittle).

\section{Related Work}

\subsection{Collective Transport Strategies for Robotic Swarms}

Various collective transport approaches have been designed to assemble large robot teams around objects that are difficult to move. However, the team sizes in such approaches are generally unpredictable, and there is the possibility of a deadlock occurring when no loads accumulate enough robots for movement but the pool of free robots is completely depleted. Our focus in this work is on the synthesis of robot control policies for collective transport 
that: (a) are derived from a dynamical model of the system, enabling theoretical analysis of the transport behavior; (b) allow robots to detach from the payload, dynamically allocating themselves to the transport team; and (c) do not require a centralized coordinator for any information other than high-level task specifications, such as the types of payloads to be retrieved.

Thus far, collective transport strategies that are designed for robotic swarms have not yet addressed scenarios with all three of these properties. Rubenstein et al (2013) derive and experimentally validate a physics-based model for transport of arbitrarily shaped rigid objects by a fixed group of robots. A dynamical model is also derived by Stilwell and Bay (1993) for the control of robots that can arrange themselves in a fixed team underneath a palletized load and use a leader-follower transport strategy. Other collective-transport strategies for fixed robot teams have employed algorithms for negotiating the direction of transport in environments with obstacles (Ferrante et al 2013) and for tuning the parameters of neural network controllers using artificial evolution (Groß and Dorigo 2009). In several strategies, load transport is performed by a subset of the swarm that changes composition over time; these strategies are investigated through simulations and experiments. O'Grady et al (2009) consider the case in a self-assembly application where broken robots themselves become immobile loads that must be removed by functional teammates. Those teammates must accumulate around immobile loads until a large enough team can move the object. Moreover, the robots must randomly leave these teams to prevent deadlock conditions. An approach to box pushing is described by Kube and Bonabeau (2000) in which robots can leave the transport team and reposition themselves on the load, switching between simple behaviors in response to locally sensed cues. Similarly, Chen et al (2013a) organize a set of pushing robots behind a tall object by taking advantage of the object's ability to block visual contact with a destination marker. In other approaches, the robots do not deliberately approach the load and propel it toward a specific location but rather affect transport through their contact with the load under the influence of an external control input. The mechanism of granular convection is exploited by Sugawara et al (2012) to drive a load to a target destination through a swarm of randomly moving robots that experience a repulsive force from the destination. Becker et al (2013) use a broadcast control input to steer the swarm around the environment and push an object encountered by the mass of robots to a target configuration.

\subsection{Control Strategies for Robotic Swarms with Stochastic Behaviors}

A variety of approaches have been developed for controlling task allocation, assembly, and self-assembly in robotic swarms with stochastic behaviors (e.g., Correll and Martinoli 2004; Berman et al 2009; Odhner and Asada 2010; Mather and Hsieh 2011; O'Grady et al 2009; Correll 2008; Matthey et al 2009; Napp et al 2009; Liu and Winfield 2010). These control strategies achieve target equilibrium populations by making use of internal timer events that may be tuned to specific parameters of the environment. For example, mobile robots that perform random walks throughout a domain will randomly encounter certain features (e.g., other robots, regions of interest, assembly components, payloads) at an average rate determined by environmental parameters. A stochastic task-allocation strategy will allocate a robot to a task according to some designed probability. Once allocated, the robot will continue to perform the task until an internally generated timeout event occurs and the robot returns to its search. If the allocation probability and return-to-search timeout are properly tuned, then the swarm of robots will converge to some desired allocation across task types in the environment. At that equilibrium, the flux of robots into each task type will match 
the flux of robots timing out of that task type. However, if the task-type encounter rates change in the environment (e.g., due to battery-related speed changes or a change in task distribution), then the timeouts will also have to be changed in order to maintain the same allocation levels. Thus, unlike our control design method, these stochastic control strategies for robotic swarms are not robust to changes in the environment.

Similar to our method, prior work has addressed the design of stochastic robot control policies for task allocation based on encounter-triggered robot behaviors. However, that prior work either deals with scenarios where encountered objects are small relative to the robot (Martinoli et al 2004; Labella et al 2006) or where large stationary objects are circumnavigated, but not manipulated, by the robots (Correll and Martinoli 2004). In contrast to this work, we address a stochastic coverage scenario in which robots physically interact with a large, moving object. As in our approach to robot controller synthesis, the task allocation strategies used by Martinoli et al (2004) and Correll and Martinoli (2004) are derived using macroscopic population models. The utility of population models in these works hinges on the ability to accurately determine the non-tunable rates of random robot encounters with objects. However, encounter rates can often be determined only through simulation (Hutchinson and Waser 2007; Gurarie 2008) because the robot motion pattern and sensor footprint and the environment configuration can induce unpredictable interacting spatial effects. Encounter rate approximations based on geometric parameters were used by Martinoli et al (2004) and Correll and Martinoli (2004), but these formulas can be applied only for environments with low densities of robots and objects where robots are uniformly spatially distributed at all times. Our load coverage scenarios violate the low-density assumption because the encountered objects, defined as zones of the loads, are adjacent to one another. Consequently, we have developed a novel control design method that can achieve target allocation levels without knowledge of the encounter rates. If absolute encounter rates are needed, for instance in designing robot policies for other applications, then we can use measurements of the system convergence time to equilibrium to infer their values.

\subsection{Relationship to Adsorption Processes}

Adsorption describes the process of particles binding to a surface for an amount of time that varies with thermodynamic parameters of the system (i.e., temperature, density, and pressure). The resulting equilibrium distribution of particles on the surface also varies with these thermodynamic properties. In our collective transport strategy, the mechanism by which robots allocate to loads is patterned in part on both reversible processes characterizing Langmuir adsorption (Langmuir 1918) and irreversible processes characterizing random sequential adsorption (RSA) (Evans 1993; Talbot et al 2000). If we used a stochastic strategy that relies on internal timer events, similar to the existing strategies described in Sect. 5.2, then the result would be an artificial adsorption process in which the equilibrium team sizes vary with the swarm size and number of loads. This work and our earlier work (Pavlic et al 2013, 2014) show that these density-dependent effects can be eliminated by mimicking far-fromequilibrium irreversible processes rather than depending on timeouts.

In Langmuir adsorption, finite-sized particles collide with a surface, bind (adsorb) to the surface with some probability, and then spontaneously unbind (desorb) later after some mean residence time. The system reaches thermodynamic equilibrium, where the flux of binding particles is balanced by the flux of unbinding particles. As the thermodynamic properties of the free particles change (e.g., as their temperature or density increases), the equilibrium allocation of particles on the surface changes. Langmuir processes have been used to design 
advanced drug delivery devices (Wang and Dormidontova 2012) which selectively target some tissues (e.g., cancers) but have negligible allocations around others (e.g., normal tissue). However, because such allocations are achieved by thermodynamic equilibrium, there must be tight control over the number of devices and knowledge of the thermodynamic variables around the tissues.

In the case of RSA, the desorption process happens at such a relatively large time scale that adsorption is considered to be irreversible. That is, particles adsorb rapidly but then take much longer to desorb, yielding the appearance or approximation that only adsorptions are occurring on random locations on the surface. Consequently, the modeled RSA system is always far from its thermodynamic equilibrium, where adsorptions and desorptions happen on a closer timescale, and instead saturates at some suboptimal packing around the surface. Rényi (1958) originally showed that the limiting fraction of a line covered with such sequentially attaching particles is a mathematical constant near 0.75 that has since been called the parking constant (Solomon and Weiner 1986; Finch 2003). Likewise, our approach cannot be used for target allocation ratios above 0.75 . However, by implementing classical RSA with a second irreversible process that catalyzes desorption (which is otherwise absent), we are able to stabilize any target allocation ratio between 0 and the parking constant. As a result, we are able to achieve a continuum of dynamical equilibria without the limitations of a thermodynamic equilibrium. This modification of RSA is trivial to implement in robotic systems with a modicum of agency.

\section{Conclusions and Future Work}

In this paper, we have applied our previous work on stochastic allocation of robotic swarms around boundaries (Pavlic et al 2013, 2014) to mimic collective-transport data obtained from Aphaenogaster cockerelli ant teams (Kumar et al 2013). This design framework allows for controlling the equilibrium demographics of multi-robot transport teams (EPMM) as well as how fast they converge to this equilibrium (TMM). Although we focused on twosided loads, the approach could be used for achieving other robot distributions that can be approximated by further dividing a load into different regions with region-specific desired allocations. Alternatively, our design approach also allows for equilibrium robot fluxes to be controlled (RMM) instead of macroscopic demographic properties. In all three cases, the equilibrium allocations of robots are robust to environmentally-induced changes to the encounter rates, including changes in swarm size and number of loads. This robustness allowed us to develop a calibration method that provides a novel way to estimate robot encounter rates without using geometrical parameters or measurements of times between events across an ensemble of robots.

One possible criticism of the current approach is that the attachment-detachment process never "turns off." Even after reaching equilibrium team demographics, free robots continue to attach and detach at random. A feature of this approach is that the system continues to adapt to environmental changes. For instance, if more loads are added, then teams will continue to form around those loads. However, in scenarios where this is not a concern, it seems more efficient for the system to detect when the desired allocation has been reached and transition to another system-level mode (e.g., from "allocation phase" to "transport phase"). Chemical reaction networks have already been constructed to implement oscillators (Lachmann and Sella 1995; Soloveichik et al 2010), counters (Soloveichik et al 2008, 2010), and algorithms which are sensitive to the macroscopic state of the system (Soloveichik et al 2010). Therefore, it may be possible to augment our approach to either detect a 
time when the system has likely converged or to directly detect the equilibrium condition and transition to a more efficient transport behavior. In fact, there are existing CRNs implemented with irreversible reactions that achieve distributed consensus (Chen et al 2013b; Shapiro and Ran 2013). These CRNs are able to detect which chemical species is in the majority and switch all entities in the mixture to that species. Because they are implemented with irreversible reactions, the equilibrium of each trial is a true fixed point of the system (i.e., it is not a thermodynamic equilibrium that fluctuates around a mean). Although our method is built using intuition from reversible processes that reach dynamic equilibria, our underlying implementation uses irreversible reactions. Consequently, we are interested in similarly leveraging these irreversible reactions to reach truly static equilibria similar to the consensus cases.

In future work, we plan on extending these methods to control two-dimensional motion of non-circular loads that can rotate. Whereas this study focused on the allocation dynamics of robots around mobile loads, our future studies will focus on other behaviors that are needed during multi-robot transport. We are particularly interested in decentralized multirobot collision avoidance for multiple transport teams that are surrounded by free robots. We also plan to develop analytically tractable models of systems with attachment-detachment probabilities that depend on the speed of the load. Using speed-dependent probabilities, it may be possible to replace the proportional velocity controller used in this paper with a simpler constant-force behavior. Such an implementation may be more suitable for microrobots and nanorobots that derive their applied forces via externally generated fields. Finally, we plan to experimentally validate our transport control strategies on a physical multi-robot testbed to eliminate any numerical artifacts of imperfect simulation tools.

Acknowledgements Comments by Simon DeDeo regarding the philosophical implications of equilibria of irreversible processes have been helpful in framing our thoughts about this approach. Interaction with him was possible due to a workshop on Information, Complexity, and Life organized by the BEYOND Center for Fundamental Concepts in Science at Arizona State University. We are also grateful for the helpful comments of four anonymous referees. This work was supported in part by NSF award no. CCF-1012029 and NSF award no. CMMI-1363499.

\section{References}

Becker A, Habibi G, Werfel J, Rubenstein M, McLurkin J (2013) Massive uniform manipulation: Controlling large populations of simple robots with a common input signal. In: IEEE/RSJ Int'l. Conf. on Intelligent Robots and Systems (IROS), IEEE, Tokyo, Japan

Berman S, Halász Á, Hsieh MA, Kumar V (2009) Optimized stochastic policies for task allocation in swarms of robots. IEEE Trans Robot 25(4):927-937, doi: 10.1109/TRO.2009.2024997

Berman S, Lindsey Q, Sakar MS, Kumar V, Pratt SC (2011) Experimental study and modeling of group retrieval in ants as an approach to collective transport in swarm robotic systems. Proc IEEE 99(9):14701481, doi: 10.1109/JPROC.2011.2111450

Chen J, Gauci M, Groß R (2013a) A strategy for transporting tall objects with a swarm of miniature mobile robots. In: Proceedings of the 2013 International Conference on Robotics and Automation, Karlsruhe, Germany, pp 863-869, doi: 10.1109/ICRA.2013.6630674

Chen YJ, Dalchau N, Srinivas N, Phillips A, Cardelli L, Soloveichik D, Seelig G (2013b) Programmable chemical controllers made from DNA. Nat Nanotechnol 8:755-762, doi: 10.1038/nnano.2013.189

Correll N (2008) Parameter estimation and optimal control of swarm-robotic systems: a case study in distributed task allocation. In: Proceedings of the 2008 IEEE International Conference on Robotics and Automation, IEEE, Pasadena, CA, USA, pp 3302-3307, doi: 10.1109/ROBOT.2008.4543714

Correll N, Martinoli A (2004) Modeling and optimization of a swarm-intelligent inspection system. In: Proceedings of the Seventh International Symposium on Distributed Autonomous Robotics Systems (DARS 2004), Toulouse, France, pp 369-378, doi: 10.1007/978-4-431-35873-2_36 
Czaczkes TJ, Ratnieks FLW (2013) Cooperative transport in ants (Hymenoptera: Formicidae) and elsewhere. Myrmecol News 18:1-11

Evans JW (1993) Random and cooperative sequential adsorption. Rev Mod Phys 65(4):1281-1329, doi: 10.1103/RevModPhys.65.1281

Ferrante E, Brambilla M, Birattari M, Dorigo M (2013) Socially-mediated negotiation for obstacle avoidance in collective transport. In: Proc. Int'l. Symp. on Distributed Autonomous Robotic Systems (DARS), Springer, Naples, Italy, Springer Tracts in Advanced Robotics, vol 83, pp 571-583

Finch SR (2003) Mathematical Constants, Encyclopedia of Mathematics and its Applications, vol 94. Cambridge University Press

Grassé PP (1959) La reconstruction du nid et les coordinations interindividuelles chez Bellicositermes natalensis et Cubitermes $\mathrm{sp}$. la théorie de la stigmergie: essai d'interprétation du comportement des termites constructeurs. Insectes Sociaux 6(1):41-80, doi: 10.1007/BF02223791

Groß R, Dorigo M (2009) Towards group transport by swarms of robots. Int'1 Journal of Bio-Inspired Computation 1(1-2):1-13

Gurarie E (2008) Models and analysis of animal movements: From individual tracks to mass dispersal. PhD thesis, University of Washington

Hutchinson JMC, Waser PM (2007) Use, misuse and extensions of "ideal gas" models of animal encounter. Biol Rev 82(3):335-359, doi: 10.1111/j.1469-185X.2007.00014.x

Kube CR, Bonabeau E (2000) Cooperative transport by ants and robots. Robot Auton Syst 30(1-2):85-101, doi: 10.1016/S0921-8890(99)00066-4

Kumar GP, Buffin A, Pavlic TP, Pratt SC, Berman SM (2013) A stochastic hybrid system model of collective transport in the desert ant Aphaenogaster cockerelli. In: Proceedings of the 16th ACM International Conference on Hybrid Systems: Computation and Control, Philadelphia, PA, pp 119-124, doi: $10.1145 / 2461328.2461349$

Labella TH, Dorigo M, Deneubourg JL (2006) Division of labor in a group of robots inspired by ants' foraging behavior. ACM Trans Auton Adapt Syst 1(1):4-25, doi: 10.1145/1152934.1152936

Lachmann M, Sella G (1995) The computationally complete ant colony: global coordination in a system with no hierarchy. In: Proceedings of the Third European Conference on Artificial Life, Granada, Spain, pp 784-800

Langmuir I (1918) The adsorption of gases on plane surfaces of glass, mica and platinum. J Am Chem Soc 40(9):1361-1403, doi: 10.1021/ja02242a004

Liu W, Winfield AFT (2010) Modeling and optimization of adaptive foraging in swarm robotic systems. Int J Robot Res 29(14):1743-1760, doi: 10.1177/0278364910375139

Martinoli A, Easton K, Agassounon W (2004) Modeling swarm robotic systems: a case study in collaborative distributed manipulation. Int J Robot Res 23(4-5):415-436, doi: 10.1177/0278364904042197

Mather TW, Hsieh MA (2011) Distributed robot ensemble control for deployment to multiple sites. In: Proceedings of Robotics: Science and Systems VII, Los Angeles, CA, USA

Matthey L, Berman S, Kumar V (2009) Stochastic strategies for a swarm robotic assembly system. In: Proceedings of the 2009 IEEE International Conference on Robotics and Automation, Kobe, Japan, pp 1953-1958

McCreery HF, Breed MD (2013) Cooperative transport in ants: a review of proximate mechanisms. Insectes Sociaux 61(2):99-110, doi: 10.1007/s00040-013-0333-3

Napp N, Burden S, Klavins E (2009) Setpoint regulation for stochastically interacting robots. In: Proceedings of Robotics: Science and Systems V, Seattle, WA, USA

Odhner LU, Asada H (2010) Stochastic recruitment control of large ensemble systems with limited feedback. J Dyn Syst, Meas, Control 132(4):041008, doi: 10.1115/1.4001706

O'Grady R, Pinciroli C, Groß R, Christensen AL, Mondada F, Bonani M, Dorigo M (2009) Swarm-bots to the rescue. In: Proceedings of the 10th European Conference on Artificial Life, Springer-Verlag, Budapest, Hungary, Lecture Notes in Computer Science, vol 5777, pp 165-172, doi: 10.1007/978-3-642-21283-3 21

Pavlic TP, Wilson S, Kumar GP, Berman S (2013) An enzyme-inspired approach to stochastic allocation of robotic swarms around boundaries. In: Proceedings of the 16th International Symposium on Robotics Research (ISRR 2013), Singapore

Pavlic TP, Wilson S, Kumar GP, Berman S (2014) Control of stochastic boundary coverage by multi-robot systems. ASME Journal of Dynamic Systems, Measurement and Control Accepted

Rényi A (1958) On a one-dimensional problem concerning random space-filling. Publ Math Inst Hung Acad Sci 3:109-127

Rubenstein M, Cabrera A, Werfel J, Habibi G, McLurkin J, Nagpal R (2013) Collective transport of complex objects by simple robots: theory and experiments. In: Proceedings of the 2013 International Conference on Autonomous Agents and Multi-Agent Systems, Saint Paul, Minnesota, USA, URL 
http://dl.acm.org/citation.cfm?id=2484920.2484932

Shapiro E, Ran T (2013) DNA computing: molecules reach consensus. Nat Nanotechnol 8:703-705, doi: 10.1038/nnano.2013.202

Solomon H, Weiner H (1986) A review of the packing problem. Commun in Stat - Theory Methods 15(9):2571-2607, doi: 10.1080/03610928608829274

Soloveichik D, Cook M, Winfree E, Bruck J (2008) Computation with finite stochastic chemical reaction networks. Nat Comput 7:615-633, doi: 10.1007/s11047-008-9067-y

Soloveichik D, Seelig G, Winfree E (2010) DNA as a universal substrate for chemical kinetics. Proc Natl Acad Sci USA 107(12):5393-5398, doi: 10.1073/pnas.0909380107

Stilwell D, Bay J (1993) Toward the development of a material transport system using swarms of ant-like robots. In: Proceedings of the International Conference on Robotics and Automation (ICRA), pp 766771, doi: 10.1109/ROBOT.1993.292070

Sugawara K, Reishus D, Correll N (2012) Object transportation by granular convection using swarm robots. In: Distributed Autonomous Robotic Systems, Springer-Verlag, Baltimore, MD, STAR, URL http://spot.colorado.edu/ dure7259/papers/Sugawara12.pdf

Talbot J, Tarjus G, Van Tassel PR, Viot P (2000) From car parking to protein adsorption: an overview of sequential adsorption processes. Colloids Surfaces, A: Physicochem Eng Asp 165(1-3):287-324, doi: 10.1016/S0927-7757(99)00409-4

Wang S, Dormidontova EE (2012) Selectivity of ligand-receptor interactions between nanoparticle and cell surfaces. Phys Rev Lett 109:238,102

Wilensky U (1999) NetLogo. Center for Connected Learning and Computer-Based Modeling, Northwestern University, Evanston, IL, USA, URL http://ccl.northwestern.edu/netlogo/

Zhang G, Fricke G, Garg D (2013) Spill detection and perimeter surveillance via distributed swarming agents. IEEE/ASME Transactions on Mechatronics 18(1):121-129, doi: 10.1109/TMECH.2011.2164578 